\title{
TaqMan Array Cards enable monitoring of diverse enteric pathogens across
} environmental and host reservoirs

Rachael Lappan ${ }^{1,2^{*}}$, Rebekah Henry ${ }^{3 *}$, Steven L. Chown ${ }^{2}$, Stephen P. Luby ${ }^{4}$, Ellen E. Higginson ${ }^{5}$, Lamiya Bata $^{3}$, Thanavit Jirapanjawat ${ }^{1,2}$, Christelle Schang ${ }^{3}$, John J. Openshaw ${ }^{4}$, Joanne O'Toole ${ }^{6}$, Audrie Lin ${ }^{7}$, Autiko Tela ${ }^{8}$, Amelia Turagabeci ${ }^{8}$, Tony H.F. Wong ${ }^{9}$, Matthew A. French ${ }^{10}$, Rebekah R. Brown ${ }^{10}$, Karin Leder $^{6}$, Chris Greening ${ }^{1,2 \#}$, David McCarthy ${ }^{3 \#}$

1. Department of Microbiology, Biomedicine Discovery Institute, Monash University, Clayton, VIC 3800, Australia

2. School of Biological Sciences, Monash University, Clayton, VIC 3800, Australia

3. Department of Civil Engineering, Monash University, Clayton, VIC 3800, Australia

4. Division of Infectious Diseases and Geographic Medicine, Stanford University, Stanford, CA 94305, USA

5. Cambridge Institute for Therapeutic Immunology and Infectious Disease, University of Cambridge, Cambridge CB2 OAW, United Kingdom

6. School of Epidemiology and Preventive Medicine, Monash University, Melbourne, VIC 3004, Australia

7. Division of Epidemiology and Biostatistics, School of Public Health, University of California Berkeley, CA 94720, USA

8. School of Public Health, Fiji National University, Suva, Fiji

9. Water Sensitive Cities Institute, Monash University, Clayton, VIC 3800, Australia

10. Monash Sustainable Development Institute, Monash University, Clayton, VIC 3800, Australia

*Co-first authors

\# Co-corresponding authors (chris.greening@monash.edu, david.mccarthy@monash.edu) 


\section{Abstract}

\section{Background}

Multiple bacteria, viruses, protists, and helminths cause enteric infections that greatly impact human health and wellbeing. These enteropathogens are transmitted via several pathways through human, animal, and environmental reservoirs. Individual quantitative PCR (qPCR) assays have been extensively used to detect enteropathogens within these types of samples, whereas the TaqMan Array Card (TAC) that allows simultaneous detection of multiple enteropathogens has only previously been validated in human clinical samples.

\section{Methods}

Here, we performed a comprehensive double-blinded comparison of the performance of a custom TAC relative to standard qPCR for the detection of eight enteric targets, by using spiked samples, wastewater from Melbourne (Australia), and human, animal, and environmental samples from informal settlements in Suva, Fiji.

\section{Findings}

Both methods exhibited high and comparable specificity (TAC: 100\%, qPCR: 94\%), sensitivity (TAC: 92\%; qPCR: 100\%), and quantitation accuracy (TAC: 91\%; qPCR: 99\%) in non-inhibited sample matrices. PCR inhibitors substantially impacted detection via TAC, though this issue was alleviated by 10 -fold sample dilution. Among samples from informal settlements, the two techniques were comparable for detection ( $89 \%$ agreement) and quantitation $\left(R^{2}=0.82\right)$. The TAC additionally included 38 other targets, enabling detection of diverse faecal pathogens and extensive environmental contamination that would be prohibitively labour intensive to assay by standard qPCR.

\section{Interpretation}

Overall, the two techniques produce comparable results across diverse sample types, with qPCR prioritising greater sensitivity and quantitation accuracy, and TAC trading small reductions in these for a cost-effective larger enteropathogen panel that enables a greater number of enteric pathogens to be analysed concurrently, which is beneficial given the abundance and variety of enteric pathogens in environments such as urban informal settlements. The ability to monitor multiple enteric pathogens across diverse reservoirs in turn allows better resolution of pathogen exposure pathways, and the design and monitoring of interventions to reduce pathogen load.

\section{Funding}

Wellcome Trust Our Planet, Our Health program [OPOH grant 205222/Z/16/Z]. 


\section{Introduction}

Diarrhoeal disease due to inadequate sanitation and poor water quality is a major public health issue and the target of one of the United Nations Sustainable Development Goals (SDG 6). This problem disproportionately affects lower- and middle-income countries, especially people living in urban informal settlements. ${ }^{1,2}$ Approximately 500,000 children under the age of five die from diarrhoeal disease each year, ${ }^{3-5}$ despite the potential to prevent an estimated 360,000 child deaths by improvements to water, sanitation and hygiene (WASH). ${ }^{6}$ Various nondiarrhoeal pathogens, most notably helminths, also contribute to enteric disease burden and malnutrition ${ }^{7}$. Moreover, asymptomatic or subclinical carriage of various enteropathogens also impacts child growth. ${ }^{8}$ Recent evidence has suggested that traditional household level WASH interventions such as pit latrines, handwashing with soap, and chlorination of water deliver suboptimal reductions in enteric disease in environments that are densely populated, ${ }^{9}$ highly contaminated ${ }^{10}$ or have a high prevalence of diarrhoea. ${ }^{11}$ This is likely due to the inability of these interventions to address the many pathways that connect environmental enteropathogens to community residents. Humans, animals, and their surrounding environments can serve as extensively interconnected reservoirs for enteropathogens. Thus, unified 'One Health' and 'Planetary Health' approaches are needed to identify pathogen exposure pathways and inform interventions that reduce pathogen load in the environment and in turn reduce human exposure. ${ }^{12}$

Assessing the extent of enteropathogen contamination and the impact of new mitigating interventions in urban informal settlements requires methods that can monitor several enteropathogen species in a range of sample types. Screening for a large number of enteropathogens is important, as multiple viruses, bacteria, protists and helminths are responsible for poor gastrointestinal health and diarrhoeal disease ${ }^{13,14}$ and mixed infections are common. ${ }^{14}$ Additionally, the relative contribution of individual pathogens to disease burden varies across settlements, within a settlement over time, and between individuals. Interventions can also disrupt some transmission pathways more effectively than others. ${ }^{10}$ The catch-all approach has traditionally been challenging due to the large number of possible enteropathogens, with a simpler solution to rely on bacterial indicator organisms to identify faecal contamination. ${ }^{15,16}$ However, faecal indicators do not correlate well with pathogen abundance and distribution, ${ }^{17-20}$ and reliance on indicators misses the complexities of enteropathogen diversity and pathogen-specific impacts of an intervention. Thus, the development of high-throughput molecular methods for enteropathogen screening of human, animal, and environmental samples would remove the need to rely solely on faecal indicators and can provide a comprehensive view of enteropathogen sources and diversity.

TaqMan quantitative PCR ( $\mathrm{PPCR}$ ) is a standard technique used across the human, animal, and environmental health fields to detect and quantify pathogens based on amplification of a pathogenspecific gene sequence..$^{19,21-23}$ This technique can be readily used to quantify pathogenic bacteria, viruses, protists, and helminths in situ, whereas alternative approaches such as selective cultivation, amplicon sequencing, and metagenomic sequencing are variably challenging to implement for nonbacterial targets. Moreover, given the ability to multiplex qPCR reactions and use 96-well and 384well plates to process many samples at a time, this technique is relatively efficient, cheap, and highthroughput with respect to sample numbers. However, the price and labour time for screening many samples for several pathogens can become high, given each additional pathogen target adds to the cost of reagents, sample volume used, and preparation time. This can become prohibitive for enteropathogen detection across human, animal, and environmental samples, where the number and taxonomic diversity of enteropathogens contributing to the burden of disease may be high and is often unknown. ${ }^{24}$ 
The TaqMan Array Card (TAC), manufactured by Applied Biosystems, is a microfluidic card designed to automate several TaqMan qPCR assays per sample. Originally designed for gene expression experiments, TAC has been effectively repurposed for detection of large panels of pathogens, ${ }^{25}$ with successful application to human faecal, ${ }^{26}$ blood, ${ }^{27}$ cerebrospinal fluid, ${ }^{28}$ and nasopharyngeal ${ }^{29}$ samples. Generally, the ability to efficiently detect large numbers of pathogens simultaneously is accompanied by a loss of sensitivity compared to standard $\mathrm{qPCR},{ }^{30,31}$ though it is highly cost effective compared to standard qPCR for the breadth of targets that can be detected. Several large multi-centre studies have used TAC to study the aetiology of diarrhoeal disease. ${ }^{14,32}$ However, TAC has rarely been applied to non-human samples, with only two studies to date using TAC to detect enteropathogens in food ${ }^{33}$ and environmenta ${ }^{18}$ samples. The latter study showed that TAC can detect a wide range of pathogens in soil and water samples from informal settlements in Kisumu, Kenya. ${ }^{18}$ However, the sensitivity, specificity, and quantitation accuracy of TAC has not yet been extensively evaluated in environmental samples in relation to the gold standard of qPCR. Thus, it is currently unclear whether the technique presents a valid alternative to standard QPCR to monitor multiple enteropathogens across different reservoirs.

In this study, we designed and evaluated a custom enteropathogen TAC that detects 46 different pathogen marker and faecal indicator genes. We first comprehensively tested the specificity, sensitivity, and accuracy of standard QPCR and TAC on a set of mock samples consisting of spiked enteropathogen genomic DNA in different sample matrices varying in PCR inhibition levels. We additionally tested both techniques on wastewater samples from Melbourne (Australia) and human stool, animal scat, environmental water, potable water, and soil samples from informal settlements in Suva, Fiji, where the diversity and prevalence of enteropathogens was not previously known. Through this approach, we demonstrate that TAC enables the reliable monitoring of multiple enteropathogens across environmental and host reservoirs. 


\section{Materials and Methods}

\subsection{Spiked sample preparation and matrix testing}

Two sets of mock samples were prepared by spiking synthetic gene blocks (IDT, Australia) representing eight enteropathogen genes (Table 1; Table S1). Dilution of gene blocks for addition to each test matrix was conducted based on conversion of the measured nanograms of resuspended amplicon to total gene copy number using the formula:

number of copies (molecules) $=\frac{\mathrm{Xng} * 6.0221 \times 10^{23} \mathrm{molecules} / \mathrm{mole}}{(\mathrm{N} * 660 \mathrm{~g} / \mathrm{mole}) * 1 \times 10^{9} \mathrm{ng} / \mathrm{g}}$

where $\mathrm{X}$ is the amount of measured amplicon (ng), $\mathrm{N}$ is the total length of dsDNA amplicon and 660 $\mathrm{g} /$ mole represents the average mass of $1 \mathrm{bp}$ dsDNA. Set 1 consisted of 10 nuclease-free water samples for comparison of method sensitivity and specificity. Three samples contained all eight targets at low (10 copies/ $\mu \mathrm{l})$, medium (100 copies/ $\mu \mathrm{l})$, or high (1000 copies/ $\mu \mathrm{l})$ concentration; six samples contained combinations of targets and concentrations; and one sample was a blank with no targets spiked.

Set 2 consisted of previously extracted Australian samples from different matrices to test the performance of the two methods under varying levels of PCR inhibition. The 36 samples tested included: nine wastewater samples with no gene blocks spiked; seven potable water samples spiked with 200 copies/ $\mu$ l of each target; and five different combinations of low, medium and high spiked targets in extracted DNA from each of four additional matrices (creek water, human stool, sediment and DNA extraction blank). All standard qPCR assays were performed on undiluted purified genomic DNA. As initial testing indicated that TAC reactions were inhibited for the wastewater and potable water matrices, these samples were assayed with TAC undiluted, diluted 1:10, and diluted 1:20 with nuclease-free water. Full details of the mock samples can be found in Table S2. All spiked samples were double-blinded and two separate laboratories delivered the results; one for TAC and another for standard qPCR.

\subsection{Fiji sample collection and processing}

Samples of child stool $(n=60)$, animal scats $(n=17)$, soil $(n=24)$, potable water $(n=10)$, and environmental water $(n=10)$ were collected from informal settlements in Suva, Fiji as part of the Revitalising Informal Settlements and their Environments (RISE) program (https://www.riseprogram/org), a transdisciplinary research program and randomised controlled trial focused on improving environmental and human health in urban informal settlements of Fiji and Indonesia. ${ }^{34,35}$

Sixty child ( $<5$ year-old) stool samples were randomly selected for the current study from a total of 287 samples collected from 12 informal settlements during the period September 27 to November 8 2019. Samples were collected by the caregiver and stored at $4^{\circ} \mathrm{C}$ on frozen gel packs prior to transport to the laboratory and storage at $-80^{\circ} \mathrm{C}$ within $24-48$ hours.

Twenty water samples were collected in clean, source-water rinsed disposable bottles from the associated settlement. Potable water was run from local municipal water sources for 1 min prior to direct collection of $2 \mathrm{~L}$ of sample. Riverine, freshwater and stormwater (environmental water) samples were taken perpendicular from the bank and at an approximate depth of $0.15 \mathrm{~m}$ at each location. For each potable and environmental water sample, $1 \mathrm{~L}$ was filtered where possible through five $0.22 \mu \mathrm{M}$ filters (Millipore). Where sediment prevented the passing of $1 \mathrm{~L}$, a reduced volume was filtered until a total of five filters were collected. Filters were stored at $-80^{\circ} \mathrm{C}$ within food-grade sealable bags. 
Soil samples were collected using a sterile tongue depressor to transfer $2 \mathrm{~cm}^{3}$ of material into foodgrade sealable bags. Total animal stools were collected and stored in the same manner, with visual assessment of stool age to prevent collection of older "dry" samples. Material was placed at $4^{\circ} \mathrm{C}$ and transferred to the laboratory within two to four hours of collection. Animal scats and soil samples were homogenised (Stomacher 400 circulator, Seward) for $1 \mathrm{~min}$ at $250 \times \mathrm{rpm}$ and stored in $0.25 \mathrm{~g}$ aliquots in sterile cryo-storage tubes at $-80^{\circ} \mathrm{C}$.

For child stool, animal scats and soil samples, total genomic DNA was isolated from $0.25 \mathrm{~g}$ of material using the QIAGEN DNeasy PowerSoil Pro kit as per manufacturer's instructions, and eluted in $50 \mu \mathrm{L}$ of sterile molecular grade water. For water samples, the filters were crushed within the bags and transferred to the bead tubes with disposable spatulas for extraction with the QIAGEN DNeasy PowerMax Soil kit with the following modifications: after the addition of buffer $\mathrm{C} 1$, the samples were incubated at $65^{\circ} \mathrm{C}$ with shaking for $30 \mathrm{~min}$ at $200 \mathrm{rpm}$ to lyse bacterial cells. The membranes were incubated for $10 \mathrm{~min}$ at room temperature in $1.5 \mathrm{ml}$ of nuclease-free water prior to elution in this volume. Viral RNA is co-extracted with these kits. Extracted nucleic acid samples were frozen at $-80^{\circ} \mathrm{C}$ prior to ambient transfer and refrigeration upon receipt in Melbourne, Australia. Samples were analysed by standard GPCR and TAC within one week without refreezing.

\subsection{Standard TaqMan qPCR detection}

Standard TaqMan qPCR assays were undertaken using primers and probes for eight target pathogens (Table 1) under the PCR reaction and cycling conditions (40 cycles) described in US-EPA Method 1696. ${ }^{36}$ The PCR was conducted on a Biorad CFX96 thermocycler (Biorad, USA). Standard curves were prepared using the gene blocks (Table $\mathbf{S 1})^{37}$ serially diluted 10 -fold to achieve a five-point standard curve ranging from $10^{5}$ to 10 copies/ $\mu \mathrm{L}$. Similarly, an internal amplification control gene block was diluted to a final concentration of 50 copies $/ \mu \mathrm{L}$ with 100 copies added to each $25 \mu \mathrm{L}$ reaction to indicate PCR inhibition.

Each $25 \mu \mathrm{L}$ reaction contained $2 \mu \mathrm{L}$ of either diluted standard gene block or sample genomic DNA. Reactions were conducted in triplicate for each sample and standard. Six replicates of no template controls were included on each run. The Sketa22 assay described in Method 1696 was not performed as salmon testes DNA was not added prior to sample extraction. Quality control, data analysis and calculations were conducted as outlined in Method 1696 $6^{36}$ (using https://www.epa.gov/sites/production/files/2019-04/methods-1696-1697-analysis-tool_march-

2019.xltm), to ensure acceptance thresholds were met for $\mathrm{R}^{2}$ (standards), amplification efficiency $(E)$, no-template control (NTC), method blank, internal amplification control, and lower limit of quantification (LLOQ). Relative fluorescence units (RFU) analysis was conducted to ensure a peak had been generated for each target assay.

\subsection{TaqMan Array Card detection}

The custom TaqMan Array Card (TAC, Applied Biosystems) contained 48 singleplex assays (Figure S1; Table S3), including the eight primer and probe sets used in the standard qPCR assays and the manufacturer's $18 \mathrm{~S}$ rRNA control. Of the 47 custom assays, 40 have previously been validated on $\mathrm{TAC}^{26,38}$ and the remaining seven were assays that have previously been published as individual TaqMan qPCR assays under similar conditions..$^{14,39-41}$ Cards were loaded with $100 \mu$ l of reaction mix per port, containing $60 \mu$ l of AgPath-ID One-Step RT-PCR master mix (Applied Biosystems; $50 \mu$ l buffer, $4 \mu \mathrm{l}$ enzyme mix and $6 \mu \mathrm{l}$ nuclease-free water per port) mixed with $40 \mu \mathrm{l}$ of sample nucleic acid. ${ }^{26}$ Samples were diluted in nuclease-free water as necessary to allow a maximum of $1400 \mathrm{ng}$ total nucleic acid per port, and 8 samples were tested per card. Loaded cards were centrifuged and sealed as per 
manufacturer's instructions, and run on a QuantStudio 7 Flex instrument (Applied Biosystems) under the following cycling conditions: $45^{\circ} \mathrm{C}$ for 20 minutes, then $95^{\circ} \mathrm{C}$ for 10 minutes, followed by 45 cycles of $95^{\circ} \mathrm{C}$ for 15 seconds and $60^{\circ} \mathrm{C}$ for 1 minute..$^{26}$

To calculate gene copies per microlitre, a standard curve was generated using synthetic plasmid controls (GeneWiz) as described in Kodani and Winchell et al. (2012). ${ }^{42}$ Three plasmids were designed with primer and probe sequences included in inserts of approximately $1 \mathrm{~kb}$ each (15-20 targets per plasmid). If the primers or probe were degenerate, the sequence from the reference genome was used. All three plasmid insert sequences are in Dataset $\mathbf{S 1}$. The three plasmid controls were combined at equal concentrations and seven 10 -fold serial dilutions were used to make the standard curve (7.2 $\times 10^{6}$ to 7.2 copies per microlitre). This positive control was run in triplicate with a no-template control on each card. Cycle threshold $\left(C_{q}\right)$ values were manually adjusted where necessary as below, with $C_{q}$ values exported to calculate a linear equation per target from the three replicates in $R$ v3.6.2. ${ }^{43}$ Note that a standard curve could not be generated for the manufacturer's $18 \mathrm{~S}$ rRNA control. The lower limit of quantitation (LLOQ) was defined as the lowest dilution of the standard curve that was detectable in all three replicates. For quality control, one of the plasmids (not containing the rotavirus target) mixed with rotavirus A RNA was analysed with each new batch of master mix to test DNA polymerase and reverse transcriptase activity. A no-template control was included once every 10 cards to monitor reagent contamination.

TAC data were reviewed within the QuantStudio Real-Time PCR Software v1.3. Each multicomponent plot was manually checked for amplification, and $C_{q}$ threshold values were checked and manually adjusted per target when the automatic threshold was inappropriate. Samples with very poor amplification curves were considered negative results, and wells flagged with both BADROX and NOISE or SPIKE, or another flag indicating a substantial issue with the well were omitted from analysis. $C_{q}$ values were exported and analysed in R v3.6.2 $2^{43}$ to calculate gene copies per microlitre of original nucleic acid extract using the standard curve for each target. For the purpose of this method comparison, all assays with a genuine amplification curve, regardless of $C_{q}$ value, were considered positive.

\subsection{Sensitivity and specificity analyses}

Sensitivity and specificity for both methods were calculated using the spiked samples as follows:

Sensitivity $=\frac{\text { true positives detected }}{\text { total spiked positives }(\text { i.e.true positives }+ \text { false negatives) }}$
Specificity $=\frac{\text { true negatives detected }}{\text { total non-spiked negatives }(\text { i.e.true negatives }+ \text { false positives) }}$

To assess quantitation accuracy, the percentage of assays that quantified gene copies per microlitre within one $\log _{10}$ of the spiked amount was calculated as follows:

$\mid \log _{10}($ spiked copies $/ \mu l)-\log _{10}($ measured copies $/ \mu l) \mid<1$

For the second set of mock samples, specificity was not calculated because it was possible for spiked targets to already be present in the samples (false positives could not be determined). Additionally, samples with a background level of target detected by either method were excluded from the quantitation accuracy calculations.

\subsection{Statistical analysis}

All statistical analyses were performed in R v3.6.2. ${ }^{43}$ Cohen's $\mathrm{k}$ statistic was calculated with the kappa2() function from package irr v.0.84.1 ${ }^{44}$ to quantify agreement between standard qPCR and TAC 
sensitivity. Wilcoxon's signed-rank test was applied with continuity correction using the wilcox.test() function. $R^{2}$ values for concordance between measured $\mathrm{QPCR}$ and TAC gene copy numbers were calculated with the $\operatorname{Im}()$ function using $\log _{10}$ transformed copy numbers with a pseudocount of 1 to accommodate values of 0 . Graphics were created with ggplot2 v3.3.2. ${ }^{45}$

\section{Results}

\subsection{Assay sensitivity and specificity under ideal conditions}

General assay sensitivity and specificity was tested using synthetic gene blocks of eight pathogen markers spiked at different concentrations into nuclease-free water in ten different combinations ( 80 individual assays; Table S2 \& S4). Across all assays, the sensitivity of TAC was slightly lower (92\%) than qPCR (100\%). TAC performed well when detecting all eight targets at low concentration (10 copies/ $\mu$ l), but sometimes failed to detect targets at this concentration when others were present at high (1000 copies/ $\mu$ l) concentration. Specificity was very high for both assays, with no false positives detected via TAC (100\%) and one false positive detected by qPCR (94\%). Both methods quantified spiked targets with variable accuracy in nuclease-free water, with TAC on average underestimating target abundance by 1.73-fold and qPCR by contrast overestimated target abundance by 2.61-fold (Figure 1; Table S4). Overall, $98.75 \%$ of all qPCR results within one log of the spiked concentration, compared to $91.25 \%$ from TAC (Table 2). Most (5/7) of these differences from the TAC results were instances of low-copy targets that were not detected by TAC. It should be noted that the layout of TACs prevent the generation of a standard curve for each run and $C_{q}$ thresholds are applied independently to each card. However, minimal run-to-run variation was observed amongst the plasmid controls, with controls providing very similar $C_{q}$ values across cards; the lowest dilution, 7.2 gene copies per microlitre, was the most variable and for some targets was not consistently detected. Altogether, these findings suggest that TAC and qPCR perform comparably in inhibitor-free sample matrices.

\subsection{Assay performance in inhibited sample matrices}

The second set of test samples was used to determine the performance of each technique on samples with varying levels of PCR inhibition (Table S2 \& S4). For both methods, there was a reduction in sensitivity (77.3\% TAC and $89.2 \%$ qPCR of spiked targets detected) and quantitation accuracy $(66.7 \%$ TAC and $69.3 \%$ qPCR assays within one log of the spiked concentration) across all sample matrices (Table 2). This decrease in performance compared to samples spiked in nuclease-free water (Figure 1) suggests both methods, especially TAC, are affected by PCR inhibitors. There was nevertheless much variability in the relative performance of the two methods across different sample matrices and pathogen targets. For example, while TAC underperformed relative to qPCR in spiked fluorinated potable water samples, the converse was true for sediment samples. Likewise, while TAC detected Cryptosporidium with higher accuracy, GPCR was more sensitive and accurate for detecting Campylobacter (Table 2). TAC also detected a range of indicators and pathogens present in Melbourne sewage and stormwater samples (Figure 3). TAC was more inhibited by this sample matrix than qPCR and detected no targets (including universal $16 \mathrm{~S}$ rRNA) in five of the eight samples. However, diluting samples (1:10 and 1:20) greatly improved detection for all samples, resulting in 216-fold and 273-fold increases in the number of targets detected respectively (Table S5). These results suggest that TAC is generally, though not consistently, less sensitive and accurate than qPCR for sample matrices with high inhibitor content. In common with previous findings, ${ }^{18}$ however, sample dilution greatly reduced inhibition without compromising detection for moderately to highly abundant targets.

\subsection{Performance comparison with faecal and environmental samples from urban informal settlements in Fiji}


A set of 121 samples from informal settlements in Fiji consisting of 60 child stool, 17 animal scats (predicted to be primarily from dogs and ducks), 20 water (10 environmental, 10 potable) samples, and 24 soil samples were analysed with TAC and standard GPCR. The nature and distribution of enteropathogen contamination in this environment is relatively unknown, and this sampling effort represents an initial insight into the baseline conditions of these settlements prior to the water and sanitation intervention to be trialled by the RISE program. ${ }^{34,35}$ The full dataset containing measured gene copies per microlitre is provided in Table S6. For the eight pathogen targets assayed by both methods, the presence/absence concordance rate was high, with $89 \%$ of all assays in agreement between both methods (Cohen's $\mathrm{k}=0.619$ ) (Figure $2 \mathrm{a}$ ). Of the remaining discordant $11 \%, 7 \%$ represented a detection by QPCR that was not observed with TAC, and $4 \%$ a TAC detection missed by qPCR; this indicates that the greater overall sensitivity of qPCR does not preclude the ability for TAC to detect pathogens when GPCR does not. Only one sample (a child stool) was indicated to be significantly inhibited by the GPCR Bacteroides internal amplification control; despite this, both methods detected the Bacteroides faecal indicator.

For assays where both methods detected the target, quantitation is quite consistent with $R^{2}=0.815$ (Figure 2b). The distribution of measured quantities for targets detected by only one method is similar on both axes, indicating that both qPCR and TAC can similarly detect targets that are missed by the other method. The target quantities measured by the two methods were significantly different $(p=$ 0.00006, Wilcoxon signed rank), which was driven by instances where a target at low concentration was detected by one method and not the other (Figure $\mathbf{2 b}$ ). When considering the concordance between the techniques when a quantity was measured by both, differences in quantities were not statistically significant ( $p=0.206$, Wilcoxon signed rank).

The pathogens detected in each sample type are shown in Figure 2a. All targets were found in at least four samples. Giardia and enteropathogenic E. coli (EPEC; eae gene) were the most common of the eight targets, whereas Salmonella and Cryptosporidium were found infrequently. Reflecting the concordance rate, detections of each target in each sample type were similar. The generic faecal indicator Bacteroides was detected more often by TAC than by QPCR, especially in child stool samples. The major discrepancy in results was the reported detection of Giardia in several soil samples by qPCR (quantified at 10-100 copies per microlitre of original sample), which were not detected by TAC. It is possible that these hits are true positives that were not detected by TAC due to a combination of low Giardia levels, sample dilution, and challenging detection in a soil matrix. However, it is also possible that false positive detection underlies these issues given Giardia qPCRs accounted for the only false positive detected in the spiking study (Figure 1 ) and thus further work is required to discriminate this. Negative extraction controls were free of amplification, with the exception of a low concentration of Giardia in the animal scat control (detected by both methods) and in the soil control (detected only by qPCR).

\subsection{Detection of other pathogens by TAC}

In addition to the eight targets assayed via both methods, the custom TAC was designed to detect a range of other viral, bacterial, protist, and helminth enteropathogen targets (Figure S1; Table S3). Of the 48 targets on the card, 44 were detected at least once (39 pathogen targets, 3 faecal indicators, 2 controls); astrovirus, S. enterica serovar Typhi (Typhoid fever), Necator americanus (hookworm), and Cystoisospora belli (isosporiasis) were not detected in any sample. Overall, most samples contained a wide range of enteropathogens (Figure 3), with the exception of potable water which contained only very low concentrations of human faecal indicators. Environmental water and animal scat samples were the most rich in enteropathogens, with most samples containing more than eight pathogens and 
ten targets (Figure 3c). Somewhat fewer pathogens were detected in child stool and soil (av. 2.8 and 2.2 pathogens per sample respectively, excluding faecal indicators).

The most prevalent enteropathogens across host and environmental reservoirs were enteroaggregative $E$. coli (EAEC) and EPEC. All three target genes were commonly detected for EAEC (aaiC, aatA, aggR), whereas the eae gene was detected more frequently than bfpA for EPEC (Figure 2a). Both markers of Shigella flexneri clade 6 (O-antigen, type 3 restriction enzyme) were also present in 18 samples. ${ }^{14}$ Giardia and Blastocystis were common protists, with Giardia present at highest concentrations in human stool (Figure 2b). Amongst helminths, the large roundworm Ascaris was most common in human faecal samples, which is concordant with findings that this genus infects approximately a sixth of the world's population. ${ }^{46}$ In contrast, Ancylostoma and Trichuris were predominant in animal faeces. Few helminths were detected in soil, but those that were present had moderately high abundance (approximately 120-140 copies per ng of DNA). Viruses were less prevalent overall; rotavirus and adenovirus F were most commonly detected, primarily in environmental water, whereas norovirus GII was abundant in one stool sample (Table S6). Some other targets, notably Campylobacter pan, Entamoeba pan, Aeromonas, and Plesiomonas shigelloides, were abundant in environmental waters and other samples. However, they were infrequently found in child stool.

TAC also detected a range of faecal indicators and other marker genes. The universal bacterial marker $16 \mathrm{~S}$ rRNA was detected in most samples, absent in only one environmental water and all potable water samples. Providing an estimate of total bacterial load, $16 \mathrm{~S}$ rRNA quantities were highest in the human faecal samples and lowest in environmental water. Aside from the universal 16S rRNA assay, the most common target detected overall was human-associated Lachnospiraceae, a faecal marker detected in the majority of child stool and environmental water samples. This suggests that Lachnospiraceae is a useful faecal indicator in this population, in agreement with previous studies. ${ }^{39,47}$ CrAssphage, an abundant bacteriophage of human Bacteroides and a proposed faecal indicator, ${ }^{41,48}$ was the least common faecal indicator detected in individual stool samples, but was present in most of the environmental water samples. These reportedly human-specific faecal indicators were also detected in the animal scats, though to a lesser extent than in child stool (Figure 2). 


\section{Discussion}

373

374

375

376

377

378

379

380

381

382

383

384

385

386

387

388

389

390

391

392

393

394

395

396

397

398

399

400

401

402

403

404

405

406

407

408

409

410

411

412

413

414

415

416

417

Until now, the TaqMan Array Card has been validated for pathogen detection in human clinical samples, ${ }^{26,30,31}$ and has, to the best of our knowledge, been used in only two studies to date to detect enteropathogens in non-human samples. ${ }^{18,33}$ In the current study, to evaluate the performance of TAC compared to standard GPCR on environmental samples, we compared enteropathogen assays with both techniques using spiked samples of known concentration, wastewater samples from Melbourne (Australia), and a range of sample types collected from urban informal settlements in Fiji. We found that the performance of TAC in environmental samples was comparable to standard qPCR with respect to specificity, sensitivity, and quantitation accuracy in clean sample matrices. Nevertheless, TAC was somewhat less sensitive than standard QPCR in detecting spiked targets in matrices with variable inhibition. Both assays varied in quantitation accuracy depending on sample matrix and pathogen target, with TAC underestimating abundance by 1.13-fold and qPCR overestimating abundance by 1.48-fold on average across the entire dataset of spiked samples. The capacity of TAC to efficiently detect multiple enteropathogen targets potentially counterbalances these tradeoffs in sensitivity and accuracy, given the benefits of monitoring a large array of enteropathogens in samples from heavily contaminated environments. In addition, we show that TAC can effectively quantify enteric pathogens across a range of environmental, human, and animal reservoirs, thereby providing a unified method to monitor pathogen transmission pathways and evaluate public health interventions.

It can be expected that TAC would detect fewer targets per sample than qPCR. The smaller reaction volume for TAC (approximately $1 \mu \mathrm{l}$ compared to $20 \mu \mathrm{l}$ standard qPCRs) means there is a reduced chance that a reaction well contains a copy of a low-concentration target. Reduction in sensitivity has been observed in previous comparisons between standard qPCR and TAC. For example, Kodani et al. (2011) compared standard GPCR and TAC performance on respiratory specimens, and observed a general 10 -fold reduction in sensitivity with TAC; some assays had a greater drop in sensitivity while others were as sensitive as standard $\mathrm{qPCR} .{ }^{49}$ Subsequent studies have also reported significant reductions in sensitivity for TAC. ${ }^{30,31}$ Importantly, some of these previous standard qPCR/TAC comparisons have evaluated the performance of TAC relative to the standard qPCR, rather than a sideby-side comparison. ${ }^{31,49}$ This means that the sensitivity and accuracy of standard qPCR is assumed to be $100 \%$ (as the gold standard), whereas that for TAC is reported as the percentage of assays that agree with standard qPCR results. This may be a reasonable approach for diagnostics in clinical samples, though our tests with spiked environmental sample matrices indicated that the performance of standard qPCR is not optimal; this method overestimated target abundance overall, falsely detected Giardia in at least one sample, and performed suboptimally in some matrices (e.g. fluorinated water, sediment). By evaluating both methods independently, we provide a clearer view of how they each perform in challenging sample types and demonstrate that they remain comparable.

TAC was generally more susceptible to inhibition by PCR inhibitors than standard qPCR in both the spiked samples and Melbourne wastewater samples. However, sample dilution by 1:10 and 1:20 was sufficient to alleviate this and yield strong amplification curves for a wide range of indicators and pathogens. By contrast, we detected minimal inhibition in the stool, scat, soil, and water samples extracted from the Fiji informal settlements with DNeasy kits, as verified by a standard qPCR internal control. We recommend that environmental studies test for PCR inhibition prior to application of TAC to enable appropriate changes in sample preparation, such as dilution, or re-extraction of samples with optimised methods. For example, studies led by Baker ${ }^{18}$ and Tsai ${ }^{33}$ initially screened for inhibition with the QuantiFast pathogen kit and used 1:10 dilution to resolve it if present. Alternatively, it is possible to introduce internal amplification controls on TAC. However, we elected not to include one for this study due to the concern that the addition of control DNA would impact the detection of 
other targets, as enteropathogens in real environmental samples were likely to be at low concentrations and the reaction volume is minimal. Instead, we included a universal 16S rRNA target in addition to the manufacturer's $18 \mathrm{~S}$ rRNA control; failure of these targets to amplify indicates either substantial inhibition or minimal sample biomass, which can be discriminated by quantifying DNA through spectroscopic methods (e.g. Nanodrop, Qubit).

As both the TAC and qPCR approaches evaluated here both use the same fundamental method of TaqMan qPCR to specifically target pathogen genes of interest, both techniques are excellent options for environmental enteropathogen monitoring. As summarised in Table 3, they have different strengths and limitations depending on purpose of their applications. Standard qPCR offers greater sensitivity and the ease of running replicates to improve confidence in positive results and quantities, while TAC is capable of detecting up to 47 custom targets across 8 samples in a minimally laborious way, eliminating pipetting error, and greatly reducing the potential for assay contamination. It is, however, important to consider the limitations of both methods. An overarching caveat is that TAC is designed to provide the best overall result and will not be optimal for individual pathogens; optimisation of assays is more easily done for standard qPCR. Quantitation is ideally performed using a standard curve included on each run, with quality control measures associated with this. ${ }^{36}$ As a TAC standard curve can only occasionally be produced (requiring one whole card per replicate), the quantitation is less stringent and standard curve-related quality control measures not applicable. We demonstrated with spiked gene blocks that accurate quantitation with TAC is achievable, but the assays that target ribosomal RNA will amplify an unknown number of gene copies per organism given rRNA copy number variation. ${ }^{50}$ Moreover, as the protocol involves a universal reverse transcription step to detect RNA viruses, both gene and mRNA copies of all targets will be amplified. This makes it challenging to accurately estimate the number of organisms per gram or millilitre of original sample, but potentially boosts sensitivity. Some of the gene targets for bacterial pathogens assayed here are encoded on plasmids, which can be of high copy number and hence further compromise quantitation. Finally, an important consideration when interpreting qPCR-based pathogen detection is that it does not indicate organism viability. Persistence of DNA from non-viable pathogens in the environment is likely to vary by organism, and presence of DNA in stool samples without active clinical infection can also complicate interpretation of results. ${ }^{51}$

In the context of assessing water and sanitation interventions in low-income contexts, however, a consistent method measuring relative change over time without the need to calculate organism numbers or identify aetiology is appropriate. We also included as many enteropathogen targets as possible, with no replicates. Customising cards to include fewer targets in duplicate, for example, may improve TAC sensitivity further. Liu et al. (2013) reported that almost half of low-concentration targets spiked into stool were detected in only one of two replicates, ${ }^{26}$ which is in agreement with the variability in the lowest dilution of our TAC standard curve. The feasibility of reducing the number of targets to introduce replicates is a trade-off dependent on the research project and the samples to be screened, and it may be beneficial to have some assays also available via standard qPCR to confirm ambiguous results or the presence of inhibition. Despite these limitations, we have shown TAC to perform comparably and have several advantages over standard GPCR that encompass the ability to scale up the number of targets with minimal impact on the amount of sample required, cost per sample, risk of assay contamination and error, and overall ease-of-use.

We demonstrated that there is a diverse range of enteropathogens present in urban informal settlements in Suva, Fiji, highlighting the utility of TAC in this setting. There was a high burden of bacterial pathogens in human stools, particularly EAEC and EPEC, as well as wide range of bacterial, protist, and helminth pathogens in environmental waters and animal scats. The inclusion and 
detection of soil-transmitted helminths in both human and environmental samples on TAC is a particularly important advance, as the standard methods for detection of such pathogens in stool involve conventional microscopy (labour-intensive, subjective, and high-risk for the operator) or serology (only viable for human-derived samples). ${ }^{52}$ Despite their transmission pathway, soiltransmitted helminths were more common in human and animal faeces than in soil samples from Fiji. The soil samples taken may not have been representative of helminth-contaminated areas, or alternatively detection may have been influenced by the integrity of helminth eggs impeding DNA extraction or the difficulties of detecting pathogens with low abundance in soil (demonstrated by our mock samples) combined with the small volume of soil extracted. Regarding human faecal indicators, Lachnospiraceae seems most suitable for this population as it was detected in the majority of faecal samples. As the assay used has been validated as highly human-specific, ${ }^{39}$ its presence in animal scats suggests a close association between animals and people in these settings, or possibly misidentification of scat origin. While Bacteroides and CrAssphage were less frequently detected in individual child stools, all three indicators were common in environmental waters. Through RISE, we are conducting further studies to understand the distribution and transmission of enteropathogens in the Fiji sites, as well as the trial settlements in Makassar, Indonesia. ${ }^{35}$

\section{Conclusion}

481 Techniques that can adequately monitor a range of enteropathogens in humans, animals and the environment are required to assess water and sanitation improvements that aim to interrupt diverse transmission pathways. The use of qPCR in the form of individual assays or via the TaqMan Array Card enables direct detection of several enteropathogens in a range of sample types, bypassing reliance on faecal indicator organisms. Our study is the first to our knowledge to evaluate the performance of a custom TAC compared to standard qPCRs on human, animal, and environmental samples. We have demonstrated that, in these challenging sample matrices, TAC is comparable to standard qPCR and is a cost-effective, scalable, accurate, and easy to use alternative for multiple pathogens. Better understanding of the distribution, transmission, and impacts of a broad range of enteropathogens across environmental, human, and animal reservoirs is essential for improvements to public health towards SDGs 3 and 6 . Among various potential applications, this will be critical for informing and evaluating future water and sanitation interventions in urban informal settlements, where the nature and extent of enteropathogen contamination is poorly characterised and diverse. More broadly, this technology enables unified approaches for surveying enteropathogens in populations and environments, as well as resolving and interrupting their transmission pathways. 


\section{Footnotes}

497

498

499

500

501

502

503

504

505

506

507

508

509

510

511

512

513

514

515

516

517

518

Acknowledgements: This work was primarily funded by the Wellcome Trust "Our Planet, Our Health" grant 205222/Z/16/Z. It was also supported by an NHMRC EL2 Fellowship (APP1178715; salary for C.G.), NHMRC Senior Research Fellowship (APP1155005; salary for K.L.) and NHMRC Equipment Grant (awarded to K.L. and S.L.C.). We also thank Eric Houpt, Darwin Operario, James Platts-Mills, Bas Dutilh, Ondrej Cinek, Brett Davis, Fiona Barker, and Sean Bay for helpful discussions. We thank David Rayner for TaqMan Array Card technical support and advice, and Sarah Thomas and Julie Bines for providing rotavirus RNA for quality control purposes. We acknowledge the hard work of the RISE field and laboratory teams in Fiji and the broader team within the RISE program. Finally, we thank all study participants in RISE.

Author contributions: Different authors were responsible for study conception (S.L.C., K.L., S.P.L., D.M., R.R.B., T.W., M.F.), experimental design (D.M., C.G., R.H., R.L., K.L., S.L.C., S.P.L., E.H., M.F.), field sampling (R.H., D.M., J.O., J.J.O., S.P.L., A.T., A.T., C.S., A.L.), DNA extractions (C.G., T.J., L.B., R.H., D.M., C.S., E.H., A.L., J.J.O, J.O.), TaqMan array card setup and analysis (R.L., C.G., E.H., K.L., S.L.C., S.P.L., J.J.O), qPCR setup and analysis (R.H., D.M., C.S.), statistical analysis (R.L., C.G.), paper writing (R.L., C.G., R.H., D.M.), and critical review and approval of the manuscript (all authors).

Ethics information: Ethics review and approval was provided by participating university and local IRBs, including Monash University Human Research Ethics Committee (Melbourne, Australia; protocol 9396) and the College Human Health Research Ethics Committee (CHREC) at the Fiji Institute of Pacific Health Research (FIPHR) and College of Medicine, Nursing, and Health Sciences at Fiji National University (FNU) (Suva, Fiji; protocol 137.19). All study settlements, households, and caregivers/respondents provide informed consent.

Conflict of interest statement: The authors declare no conflict of interest. 


\section{References}

1 Riley LW, Ko Al, Unger A, Reis MG. Slum health: Diseases of neglected populations. BMC Int Health Hum Rights 2007; 7: 2.

2 Nadimpalli ML, Marks SJ, Montealegre MC, et al. Urban informal settlements as hotspots of antimicrobial resistance and the need to curb environmental transmission. Nat Microbiol 2020; 5: 787-95.

3 Liu L, Oza S, Hogan D, et al. Global, regional, and national causes of under-5 mortality in 2000-15: an updated systematic analysis with implications for the Sustainable Development Goals. The Lancet 2016; 388: 3027-35.

4 Liu L, Oza S, Hogan D, et al. Global, regional, and national causes of child mortality in 2000-13, with projections to inform post-2015 priorities: an updated systematic analysis. The Lancet 2015; 385: 430-40.

5 Kotloff KL, Nataro JP, Blackwelder WC, et al. Burden and aetiology of diarrhoeal disease in infants and young children in developing countries (the Global Enteric Multicenter Study, GEMS): a prospective, case-control study. The Lancet 2013; 382: 209-22.

6 Prüss-Ustün A, Bartram J, Clasen T, et al. Burden of disease from inadequate water, sanitation and hygiene in low- and middle-income settings: a retrospective analysis of data from 145 countries. Trop Med Int Health 2014; 19: 894-905.

7 Bethony J, Brooker S, Albonico M, et al. Soil-transmitted helminth infections: ascariasis, trichuriasis, and hookworm. The Lancet 2006; 367: 1521-32.

8 Rogawski ET, Liu J, Platts-Mills JA, et al. Use of quantitative molecular diagnostic methods to investigate the effect of enteropathogen infections on linear growth in children in low-resource settings: longitudinal analysis of results from the MAL-ED cohort study. Lancet Glob Health 2018; 6: e1319-28.

9 Bauza V, Madadi V, Ocharo R, Nguyen TH, Guest JS. Enteric pathogens from water, hands, surface, soil, drainage ditch, and stream exposure points in a low-income neighborhood of Nairobi, Kenya. Sci Total Environ 2020; 709: 135344.

10 Rogawski McQuade ET, Platts-Mills JA, Gratz J, et al. Impact of Water Quality, Sanitation, Handwashing, and Nutritional Interventions on Enteric Infections in Rural Zimbabwe: The Sanitation Hygiene Infant Nutrition Efficacy (SHINE) Trial. J Infect Dis 2020; 221: 1379-86.

11 Null C, Stewart CP, Pickering AJ, et al. Effects of water quality, sanitation, handwashing, and nutritional interventions on diarrhoea and child growth in rural Kenya: a cluster-randomised controlled trial. Lancet Glob Health 2018; 6: e316-29.

12 Whitmee S, Haines A, Beyrer C, et al. Safeguarding human health in the Anthropocene epoch: report of The Rockefeller Foundation-Lancet Commission on planetary health. The Lancet 2015; 386: 1973-2028.

13 Troeger C, Blacker BF, Khalil IA, et al. Estimates of the global, regional, and national morbidity, mortality, and aetiologies of diarrhoea in 195 countries: a systematic analysis for the Global Burden of Disease Study 2016. Lancet Infect Dis 2018; 18: 1211-28. 
14 Liu J, Platts-Mills JA, Juma J, et al. Use of quantitative molecular diagnostic methods to identify causes of diarrhoea in children: a reanalysis of the GEMS case-control study. The Lancet 2016; 388: 1291-301.

15 Pickering AJ, Ercumen A, Arnold BF, et al. Fecal Indicator Bacteria along Multiple Environmental Transmission Pathways (Water, Hands, Food, Soil, Flies) and Subsequent Child Diarrhea in Rural Bangladesh. Environ Sci Technol 2018; 52: 7928-36.

16 Holcomb DA, Knee J, Sumner T, et al. Human fecal contamination of water, soil, and surfaces in households sharing poor-quality sanitation facilities in Maputo, Mozambique. Int J Hyg Environ Health 2020; 226: 113496.

17 Korajkic A, McMinn BR, Harwood VJ. Relationships between Microbial Indicators and Pathogens in Recreational Water Settings. Int J Environ Res Public Health 2018; 15. DOI:10.3390/ijerph15122842.

18 Baker KK, Senesac R, Sewell D, Sen Gupta A, Cumming O, Mumma J. Fecal Fingerprints of Enteric Pathogen Contamination in Public Environments of Kisumu, Kenya, Associated with Human Sanitation Conditions and Domestic Animals. Environ Sci Technol 2018; 52: 10263-74.

19 Fuhrmeister ER, Ercumen A, Pickering AJ, et al. Predictors of Enteric Pathogens in the Domestic Environment from Human and Animal Sources in Rural Bangladesh. Environ Sci Technol 2019; 53: 10023-33.

20 Harwood VJ, Levine AD, Scott TM, et al. Validity of the Indicator Organism Paradigm for Pathogen Reduction in Reclaimed Water and Public Health Protection. Appl Environ Microbiol 2005; 71: 3163-70.

21 Aw TG, Rose JB. Detection of pathogens in water: from phylochips to qPCR to pyrosequencing. Curr Opin Biotechnol 2012; 23: 422-30.

22 Noble RT, Blackwood AD, Griffith JF, McGee CD, Weisberg SB. Comparison of Rapid Quantitative PCR-Based and Conventional Culture-Based Methods for Enumeration of Enterococcus spp. and Escherichia coli in Recreational Waters. Appl Environ Microbiol 2010; 76: 7437-43.

23 Ishii S, Segawa T, Okabe S. Simultaneous Quantification of Multiple Food- and Waterborne Pathogens by Use of Microfluidic Quantitative PCR. Appl Environ Microbiol 2013; 79: 2891-8.

24 Ramírez-Castillo FY, Loera-Muro A, Jacques M, et al. Waterborne Pathogens: Detection Methods and Challenges. Pathogens 2015; 4: 307-34.

25 Heaney J, Rolfe K, Gleadall NS, Greatorex JS, Curran MD. Chapter 6 - Low-Density TaqMan ${ }^{\circledR}$ Array Cards for the Detection of Pathogens. In: Sails A, Tang Y-W, eds. Methods in Microbiology. Academic Press, 2015: 199-218.

26 Liu J, Gratz J, Amour C, et al. A Laboratory-Developed TaqMan Array Card for Simultaneous Detection of 19 Enteropathogens. J Clin Microbiol 2013; 51: 472-80.

27 Moore CC, Jacob ST, Banura P, et al. Etiology of Sepsis in Uganda Using a Quantitative Polymerase Chain Reaction-based TaqMan Array Card. Clin Infect Dis 2019; 68: 266-72.

28 Zhao C, Wang X, Zhang C, et al. Development of a TaqMan Array card to target 21 purulent meningitis-related pathogens. BMC Infect Dis 2019; 19: 289. 
29 Steensels D, Reynders M, Descheemaeker P, et al. Performance evaluation of direct fluorescent antibody, Focus Diagnostics Simplexa ${ }^{\mathrm{TM}}$ Flu A/B \& RSV and multi-parameter customized respiratory Taqman ${ }^{\circledR}$ array card in immunocompromised patients. J Virol Methods 2017; 245: 615.

30 Harvey JJ, Chester S, Burke SA, et al. Comparative analytical evaluation of the respiratory TaqMan Array Card with real-time PCR and commercial multi-pathogen assays. J Virol Methods 2016; 228: $151-7$.

31 Weinberg GA, Schnabel KC, Erdman DD, et al. Field evaluation of TaqMan Array Card (TAC) for the simultaneous detection of multiple respiratory viruses in children with acute respiratory infection. J Clin Virol 2013; 57: 254-60.

32 Platts-Mills JA, Liu J, Rogawski ET, et al. Use of quantitative molecular diagnostic methods to assess the aetiology, burden, and clinical characteristics of diarrhoea in children in low-resource settings: a reanalysis of the MAL-ED cohort study. Lancet Glob Health 2018; 6: e1309-18.

33 Tsai K, Simiyu S, Mumma J, et al. Enteric Pathogen Diversity in Infant Foods in Low-Income Neighborhoods of Kisumu, Kenya. Int J Environ Res Public Health 2019; 16: 506.

34 Brown R, Leder K, Wong T, et al. Improving human and environmental health in urban informal settlements: the Revitalising Informal Settlements and their Environments (RISE) programme. Lancet Planet Health 2018; 2: S29.

35 Leder K, Openshaw J, Allotey P, et al. Study design, rationale and methods of the Revitalising Informal Settlements and their Environments (RISE) study: a cluster randomised controlled trial to evaluate environmental and human health impacts of a water sensitive intervention in informal settlements in Indonesia and Fiji. BMJ Open 2020; (submitted).

36 United States Environmental Protection Agency. Method 1696: Characterization of Human Fecal Pollution in Water by HF183/BacR287 TaqMan ${ }^{\circledR}$ Quantitative Polymerase Chain Reaction (qPCR) Assay (EPA 821-R-19-002). 2019; published online March. https://www.epa.gov/sites/production/files/2019-03/documents/method_1696_draft_2019.pdf.

37 Conte J, Potoczniak MJ, Tobe SS. Using synthetic oligonucleotides as standards in probe-based qPCR. BioTechniques 2018; 64: 177-9.

38 Liu J, Gratz J, Amour C, et al. Optimization of Quantitative PCR Methods for Enteropathogen Detection. PLOS ONE 2016; 11: e0158199.

39 Feng S, Bootsma M, McLellan SL. Human-Associated Lachnospiraceae Genetic Markers Improve Detection of Fecal Pollution Sources in Urban Waters. Appl Env Microbiol 2018; 84: e00309-18.

40 Green HC, Haugland RA, Varma M, et al. Improved HF183 Quantitative Real-Time PCR Assay for Characterization of Human Fecal Pollution in Ambient Surface Water Samples. Appl Env Microbiol 2014; 80: 3086-94.

41 Stachler E, Kelty C, Sivaganesan M, Li X, Bibby K, Shanks OC. Quantitative CrAssphage PCR Assays for Human Fecal Pollution Measurement. Environ Sci Technol 2017; 51: 9146-54.

42 Kodani M, Winchell JM. Engineered Combined-Positive-Control Template for Real-Time Reverse Transcription-PCR in Multiple-Pathogen-Detection Assays. J Clin Microbiol 2012; 50: 1057-60. 
43 R Core Team. R: A Language and Environment for Statistical Computing. Vienna, Austria: R Foundation for Statistical Computing, 2019 https://www.R-project.org/.

44 Gamer M, Lemon J, Fellows I, Singh P. irr: Various Coefficients of Interrater Reliability and Agreement. 2019 https://CRAN.R-project.org/package=irr.

45 Wickham H. ggplot2: Elegant Graphics for Data Analysis. Springer-Verlag New York, 2016 https://ggplot2.tidyverse.org.

46 Dold C, Holland CV. Ascaris and ascariasis. Microbes Infect 2011; 13: 632-7.

47 Newton RJ, VandeWalle JL, Borchardt MA, Gorelick MH, McLellan SL. Lachnospiraceae and Bacteroidales Alternative Fecal Indicators Reveal Chronic Human Sewage Contamination in an Urban Harbor. Appl Environ Microbiol 2011; 77: 6972-81.

48 Edwards RA, Vega AA, Norman HM, et al. Global phylogeography and ancient evolution of the widespread human gut virus crAssphage. Nat Microbiol 2019; 4: 1727-36.

49 Kodani M, Yang G, Conklin LM, et al. Application of TaqMan Low-Density Arrays for Simultaneous Detection of Multiple Respiratory Pathogens. J Clin Microbiol 2011; 49: 2175-82.

50 Klappenbach JA, Dunbar JM, Schmidt TM. rRNA Operon Copy Number Reflects Ecological Strategies of Bacteria. Appl Environ Microbiol 2000; 66: 1328-33.

51 Platts-Mills JA, Operario DJ, Houpt ER. Molecular Diagnosis of Diarrhea: Current Status and Future Potential. Curr Infect Dis Rep 2012; 14: 41-6.

52 Khurana S, Sethi S. Laboratory diagnosis of soil transmitted helminthiasis. Trop Parasitol 2017; 7: 86-91. 


\section{Tables}

658 Table 1. TaqMan qPCR assays used for detection by standard qPCR and custom TaqMan Array Cards.

\begin{tabular}{|c|c|c|c|c|}
\hline Organism & $\begin{array}{l}\text { Targeted } \\
\text { gene }\end{array}$ & $\begin{array}{l}\text { Forward primer } \\
\left(5^{\prime} \text { to } 3^{\prime}\right)\end{array}$ & $\begin{array}{l}\text { Reverse primer }\left(5^{\prime}\right. \\
\left.\text { to } 3^{\prime}\right)\end{array}$ & $\begin{array}{l}\text { TaqMan probe } \\
\text { (standalone qPCR) }\end{array}$ \\
\hline $\begin{array}{l}\text { Campylobacter jejuni / } \\
\text { coli }\end{array}$ & cadF & $\begin{array}{l}\text { CTGCTAAACCATA } \\
\text { GAAATAAAATTTCT } \\
\text { CAC }\end{array}$ & $\begin{array}{l}\text { CTTTGAAGGTAATT } \\
\text { TAGATATGGATAA } \\
\text { TCG }\end{array}$ & $\begin{array}{l}\text { 5'HEX- } \\
\text { CATTTTGACGATTTTTGG } \\
\text { CTTGA-3'MGB }\end{array}$ \\
\hline Salmonella enterica & invA & $\begin{array}{l}\text { TCGGGCAATTCGTT } \\
\text { ATTGG }\end{array}$ & $\begin{array}{l}\text { GATAAACTGGACC } \\
\text { ACGGTGACA }\end{array}$ & $\begin{array}{l}\text { 5'FAM- } \\
\text { AAGACAACAAAACCCAC } \\
\text { CGC-3'MGB }\end{array}$ \\
\hline $\begin{array}{l}\text { Shiga toxin-producing } \\
\text { Escherichia coli (STEC) }\end{array}$ & stx1 & $\begin{array}{l}\text { ACTTCTCGACTGCA } \\
\text { AAGACGTATG }\end{array}$ & $\begin{array}{l}\text { ACAAATTATCCCCT } \\
\text { GWGCCACTATC }\end{array}$ & $\begin{array}{l}\text { 5'Texas Red- } \\
\text { CTCTGCAATAGGTACTCC } \\
\text { A-3'MGB }\end{array}$ \\
\hline STEC & stx2 & $\begin{array}{l}\text { CCACATCGGTGTCT } \\
\text { GTTATTAACC }\end{array}$ & $\begin{array}{l}\text { GGTCAAAACGCGC } \\
\text { CTGATAG }\end{array}$ & $\begin{array}{l}\text { 5'FAM- } \\
\text { TTGCTGTGGATATACGA } \\
\text { GG-3'MGB }\end{array}$ \\
\hline $\begin{array}{l}\text { Enteropathogenic } E \text {. } \\
\text { coli (EPEC) }\end{array}$ & eae & $\begin{array}{l}\text { CATTGATCAGGATT } \\
\text { TTTCTGGTGATA }\end{array}$ & $\begin{array}{l}\text { CTCATGCGGAAAT } \\
\text { AGCCGTTA }\end{array}$ & $\begin{array}{l}\text { 5'FAM- } \\
\text { ATACTGGCGAGACTATTT } \\
\text { CAA-3'MGB }\end{array}$ \\
\hline Crypto & 18S rRNA & $\begin{array}{l}\text { GGGTTGTATTTATT } \\
\text { AGATAAAGAACCA }\end{array}$ & $\begin{array}{l}\text { AGGCCAATACCCT } \\
\text { ACCGTCT }\end{array}$ & $\begin{array}{l}\text { 5'FAM- } \\
\text { TGACATATCATTCAAGTT } \\
\text { TCTGAC-3'MGB }\end{array}$ \\
\hline Giardia & 18S rRNA & $\begin{array}{l}\text { GACGGCTCAGGAC } \\
\text { AACGGTT }\end{array}$ & $\begin{array}{l}\text { TTGCCAGCGGTGT } \\
\text { CCG }\end{array}$ & $\begin{array}{l}\text { 5'HEX- } \\
\text { CCCGCGGCGGTCCCTGC } \\
\text { TAG-3'MGB }\end{array}$ \\
\hline Bacteroides & 16S rRNA & $\begin{array}{l}\text { ATCATGAGTTCACA } \\
\text { TGTCCG }\end{array}$ & $\begin{array}{l}\text { CTTCCTCTCAGAAC } \\
\text { СССТАTCC }\end{array}$ & $\begin{array}{l}\text { 5'FAM- } \\
\text { CTAATGGAACGCATCCC- } \\
\text { 3'MGB }\end{array}$ \\
\hline $\begin{array}{l}\text { Internal amplification } \\
\text { control }^{b}\end{array}$ & 16S rRNA & $\begin{array}{l}\text { ATCATGAGTTCACA } \\
\text { TGTCCG }\end{array}$ & $\begin{array}{l}\text { CTTCCTCTCAGAAC } \\
\text { CССТATCC }\end{array}$ & $\begin{array}{l}\text { 5'VIC- } \\
\text { AACACGCCGTTGCTACA- } \\
\text { 3'MGB }\end{array}$ \\
\hline
\end{tabular}

${ }^{a}$ TaqMan Array Card probes were identical with the exception of the fluorophore (all TAC probes

660 were 5'FAM 3'MGB).

$661{ }^{\mathrm{b}}$ Internal amplification control targets Bacteroides and was applied to standalone qPCR assays only. 
662

663

664

665

666

667

Table 2. Performance of TAC and qPCR on spiked samples in sample matrices varying in levels of PCR inhibitors. Results are shown by sample matrix and by target. Sensitivity is defined as the percentage of spiked targets that were detected. Accuracy is measured as percentage of assays within one $\log _{10}$ of the spiked concentration; assays where background levels of pathogen were detected by qPCR or TAC are excluded from these calculations.

\begin{tabular}{|l|c|c|c|c|}
\hline Sample matrix & $\begin{array}{c}\text { Sensitivity } \\
\text { (TAC, \%) }\end{array}$ & $\begin{array}{c}\text { Sensitivity } \\
\text { (qPCR, \%) }\end{array}$ & $\begin{array}{c}\text { Accuracy } \\
\text { (TAC, \%) }\end{array}$ & $\begin{array}{c}\text { Accuracy } \\
\text { (qPCR, \%) }\end{array}$ \\
\hline $\begin{array}{l}\text { Nuclease-free } \\
\text { water }\end{array}$ & 92.2 & 100 & 91.3 & 98.8 \\
\hline Creek water & 93.3 & 96.7 & 80.0 & 80.0 \\
\hline Sediment & 60.0 & 56.7 & 42.5 & 30.0 \\
\hline Human stool & 83.3 & 96.7 & 80.0 & 90.0 \\
\hline $\begin{array}{l}\text { Fluorinated } \\
\text { potable water }\end{array}$ & 71.4 & 94.6 & 62.5 & 73.2 \\
\hline Extraction blank & 83.3 & 96.7 & 80.0 & 82.5 \\
\hline Target & & & & 81.5 \\
\hline Bacteroides & 85.2 & 85.2 & 85.2 \\
\hline $\begin{array}{l}\text { Campylobacter } \\
\text { jejuni / coli }\end{array}$ & 77.4 & 87.1 & 48.6 & 78.4 \\
\hline Cryptosporidium & 77.4 & 90.3 & 70.3 & 54.1 \\
\hline EPEC (eae) & 74.2 & 90.3 & 73.0 & 78.4 \\
\hline Giardia & 90.3 & 96.8 & 81.5 & 81.5 \\
\hline Salmonella & 83.9 & 96.8 & 78.1 & 68.8 \\
\hline STEC (stx1) & 85.2 & 96.3 & 87.5 & 90.6 \\
\hline STEC (stx2) & 77.4 & 93.5 & 78.4 & 91.9 \\
\hline
\end{tabular}


Table 3. Comparison of TAC vs qPCR for monitoring multiple pathogens.

\begin{tabular}{|c|c|c|}
\hline Method & $\begin{array}{l}\text { Quantitative PCR (qPCR) and reverse } \\
\text { transcriptase } q P C R \text { (RT-qPCR) }\end{array}$ & TaqMan Array Cards (TAC) \\
\hline Target range & $\begin{array}{l}\text { Narrow target range: Individually } \\
\text { detects any target of interest with } \\
\text { appropriately optimised } \\
\text { primers/probes. However, adding } \\
\text { additional targets requires extra sample } \\
\text { volume, labour, cost, and plastic waste. } \\
\text { Assays can be multiplexed (detection of } \\
\text { multiple targets in one reaction) with } \\
\text { careful optimisation. }\end{array}$ & $\begin{array}{l}\text { Broad target range: Simultaneously } \\
\text { detects up to } 47 \text { targets and } 1 \text { internal } \\
\text { control across } 8 \text { samples. Assays } \\
\text { require careful card design and } \\
\text { manufacture with appropriate lead- } \\
\text { time. Optimisation may be required for } \\
\text { target quantitation under universal } \\
\text { conditions on the card. }\end{array}$ \\
\hline $\begin{array}{l}\text { Sensitivity / } \\
\text { accuracy }\end{array}$ & $\begin{array}{l}\text { High sensitivity and accuracy: } \\
\text { Theoretical detection limit is } \\
\text { approximately three gene copies per } \\
\text { reaction. Pathogen quantitation } \\
\text { possible with appropriate reference } \\
\text { standards. PCR inhibition possible, but } \\
\text { can be readily monitored with controls. }\end{array}$ & $\begin{array}{l}\text { High sensitivity / medium accuracy: } \\
\text { Sensitivity high but often lower than } \\
\text { qPCR given smaller reaction volume } \\
\text { and universal reaction conditions. } \\
\text { Pathogen quantitation possible with } \\
\text { reference standards, but generally } \\
\text { requires comparisons between cards. } \\
\text { Quantitation also challenging due to } \\
\text { co-detection of DNA and RNA due to } \\
\text { universal reverse transcriptase step to } \\
\text { detect RNA viruses. Greater potential } \\
\text { for PCR inhibition. }\end{array}$ \\
\hline Specificity & $\begin{array}{l}\text { High specificity: Well-designed TaqMan } \\
\text { primer and probe sequences are very } \\
\text { specific. }\end{array}$ & $\begin{array}{l}\text { High specificity: Same TaqMan } \\
\text { technology as standard qPCR. }\end{array}$ \\
\hline Scalability & $\begin{array}{l}\text { Moderate scalability: Extensive manual } \\
\text { handling with large numbers of } \\
\text { samples and/or pathogens. Large } \\
\text { sample numbers require high labour } \\
\text { time or robotics. Increased sample } \\
\text { numbers require greater sample } \\
\text { volume and produce more waste. High } \\
\text { potential for pipetting errors. }\end{array}$ & $\begin{array}{l}\text { High scalability: Simple and } \\
\text { moderately fast ( } 3 \text { hours) to prepare } \\
\text { and run from extracted nucleic acids. } \\
\text { Labour time is minimal given the few } \\
\text { manual handling tasks required, } \\
\text { though increases per card (eight } \\
\text { samples). Low potential for pipetting } \\
\text { errors. }\end{array}$ \\
\hline Cost & $\begin{array}{l}\text { Low cost per sample: Low reagent cost } \\
\text { per sample (approximately USD } \$ 2.10 \\
\text { for one pathogen without replicates). } \\
\text { Small cost increase with more samples, } \\
\text { but large increase with more targets } \\
\text { (double the labour and reagents cost } \\
\text { for two targets). }\end{array}$ & $\begin{array}{l}\text { Low cost per pathogen: Moderate } \\
\text { reagent cost per sample (approx. USD } \\
\$ 60) \text {. However, highly cost effective for } \\
\text { monitoring multiple targets per sample } \\
\text { (approx. USD \$1.28 per sample per } \\
\text { target without replicates). }\end{array}$ \\
\hline Resources & $\begin{array}{l}\text { Moderate resources: Requires real- } \\
\text { time thermal cycler. Training for } \\
\text { molecular biology, equipment use, and } \\
\text { software required. }\end{array}$ & $\begin{array}{l}\text { Moderate resources: Requires real- } \\
\text { time thermal cycler with array card } \\
\text { block. Training needed for molecular } \\
\text { biology, equipment, and software. }\end{array}$ \\
\hline
\end{tabular}




\section{Figure legends}

672 Figure 1. Quantitation of spiked genetic material in nuclease-free water by TAC and standard qPCR. Ten 673 different combinations of spiked material were tested in a randomised double-blinded manner. Targets 674 were either: spiked randomly in different combinations (samples 1, 3, 4, 6, 9, 10); spiked at consistent 675 concentrations of 10 (sample 7), 100 (sample 2), or 1000 (sample 8) copies per microlitre; or not spiked 676 at all (sample 5; a blank control). For each target, the quantity of material spiked (white circle), the copies 677 detected by standard qPCR (blue circle), and the copies detected by TAC (yellow circle) are shown.

678 Figure 2. Concordance between standard qPCR and TAC in detecting pathogens in animal scats, child 679 stool, soil, and water collected from informal settlements of Suva, Fiji. Agreement between the methods 680 with respect to a) the number of positive detections of targets and $\mathbf{b}$ ) the measured target quantity in $681 \log _{10}$ gene copies per microlitre of extracted DNA (with a pseudocount of 1 added before $\log _{10}$ 682 transformation). The regression lines with associated $95 \%$ confidence intervals are shown for the subset 683 of data where a target was quantified by both methods (blue, $R^{2}=0.815$ ). Across all data points, $R^{2}=$ 6840.668 (black).

685 Figure 3. Pathogen and indicator targets detected via TaqMan Array Card in Melbourne wastewater 686 samples and animal scats, child stool, soil, and water collected from informal settlements of Suva, Fiji. Heatmaps represent the a) prevalence (percentage of positive samples) and $\mathbf{b}$ ) abundance (mean value of $\log _{10}$ gene copies per nanogram of DNA across positive samples) of each target by sample type. White represents a zero value, and 18S rRNA quantitation was unavailable. The number of pathogens or indicators detected per sample is represented by c) histograms, also by sample type. This excludes $16 \mathrm{~S}$ rRNA and $18 \mathrm{~S}$ rRNA and counts pathogens with multiple gene targets once. 
a)

Prevalence

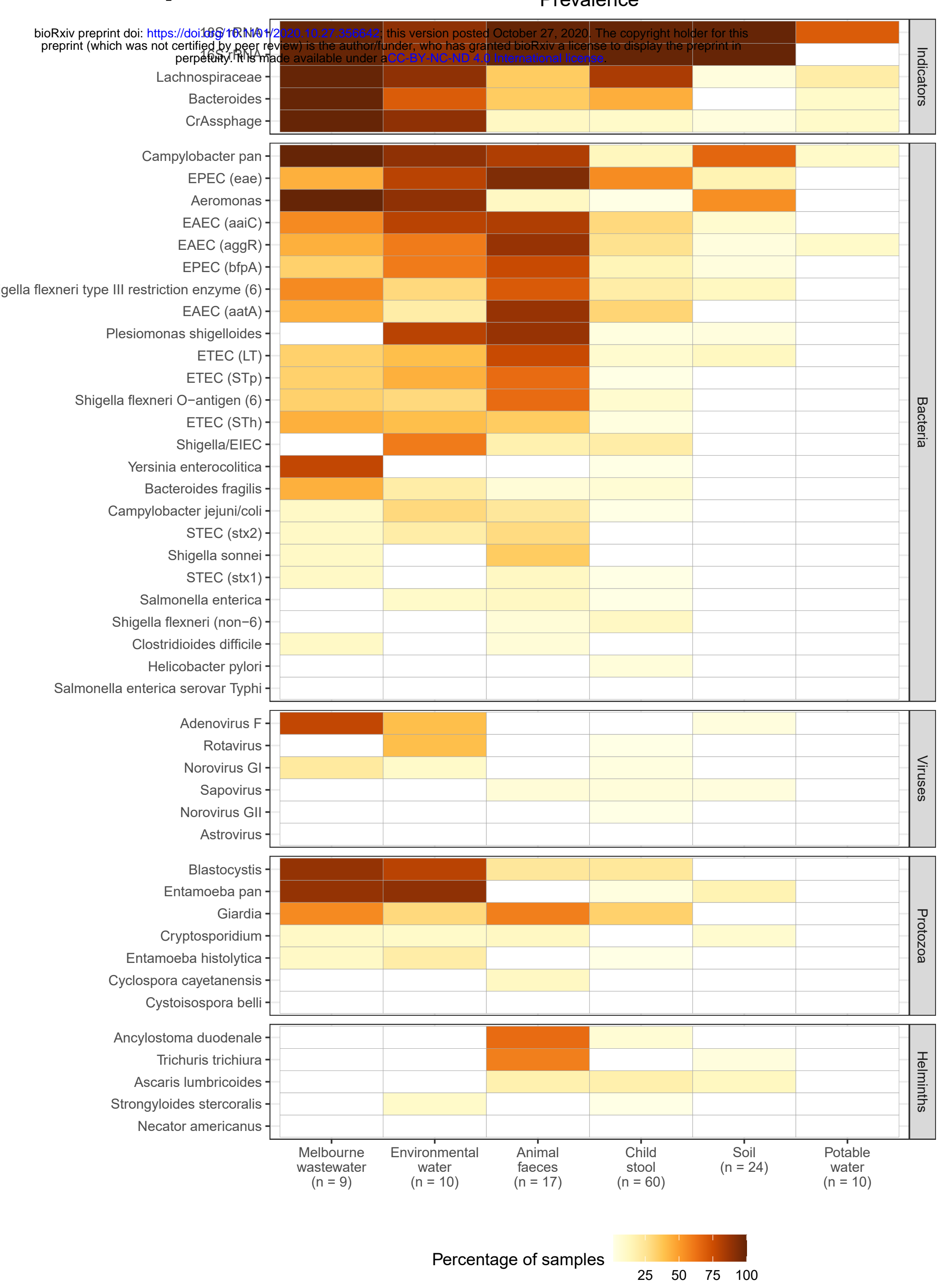

b)

Abundance

c)
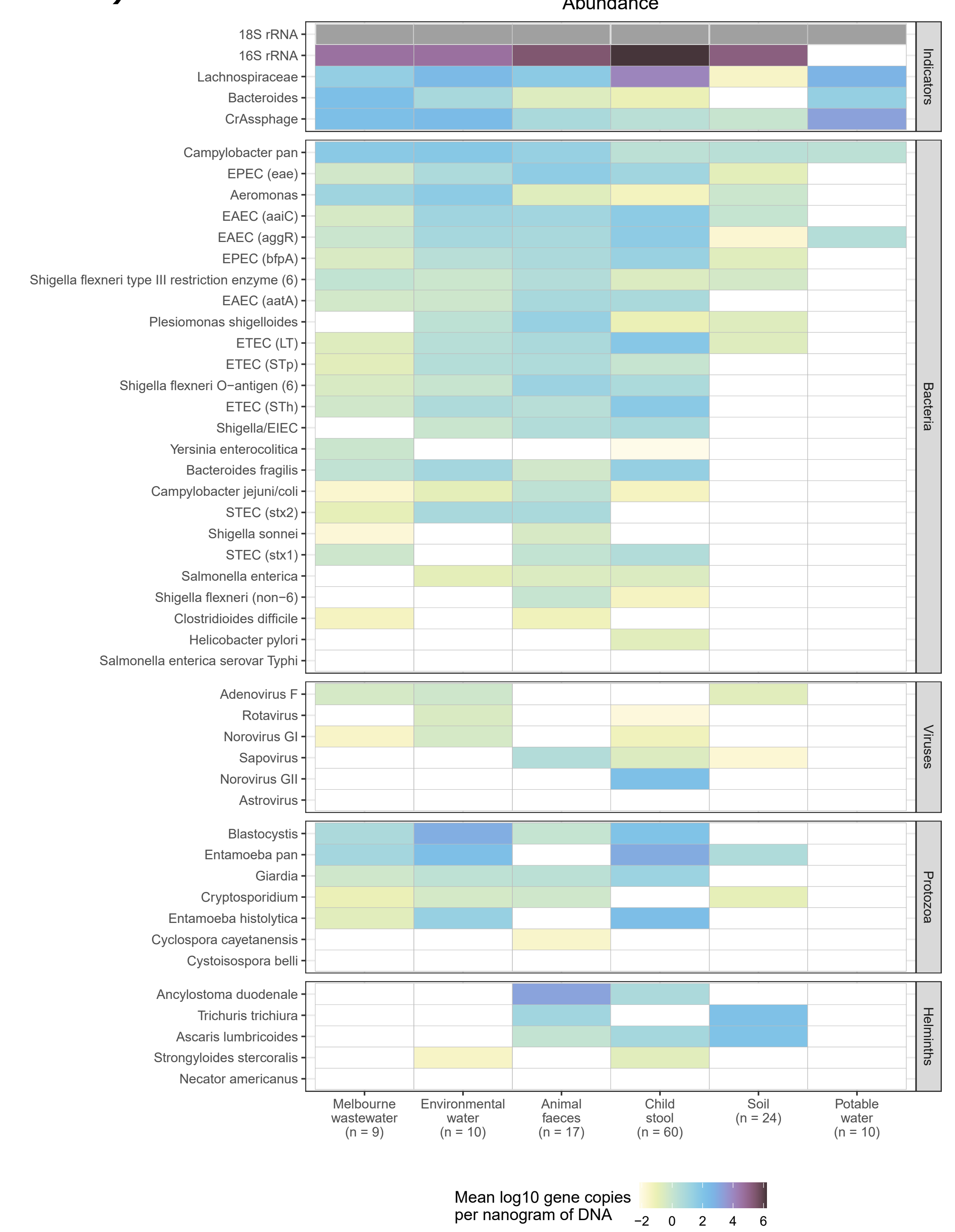

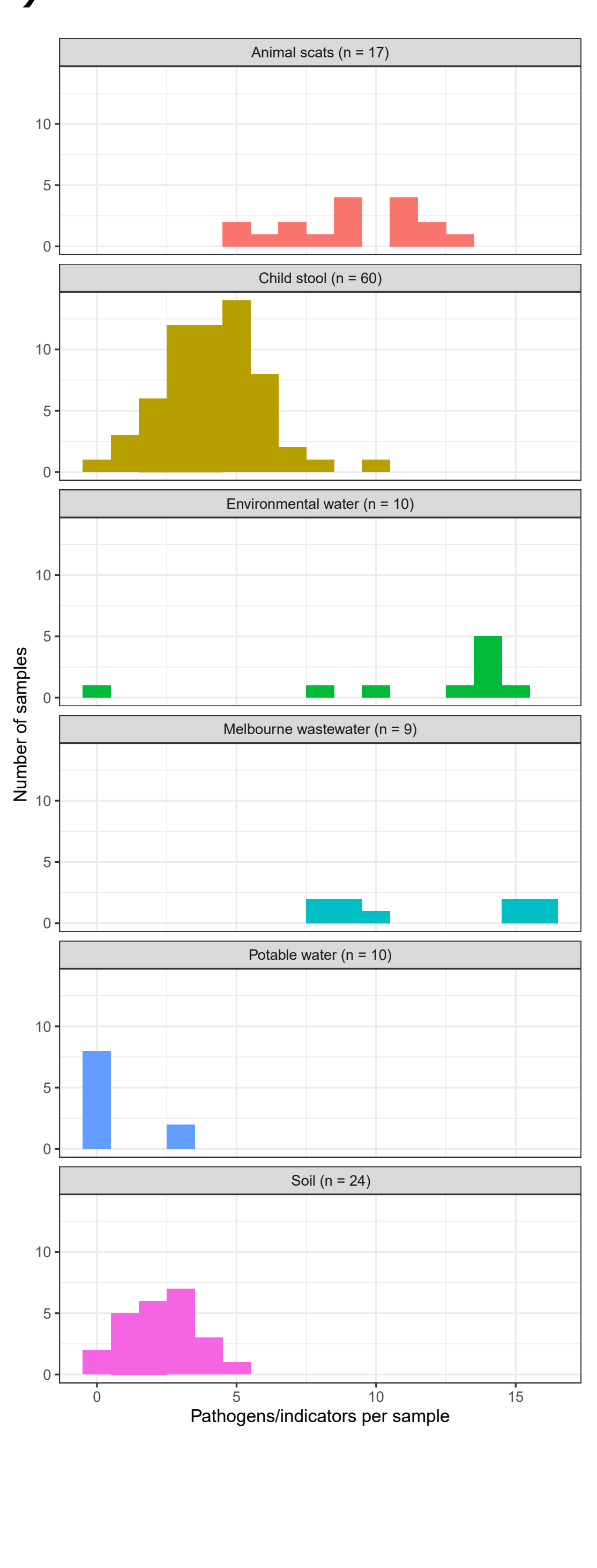

